\title{
دراسة مدى تأثير زيادة المساحة على تكاليف أعمال التشطيبات
}

\author{
Maged Moneer Gad
}

Department of Architecture- Obour High Institute of Engineering and Technology, Cairo, Egypt gadmaged@yahoo.de

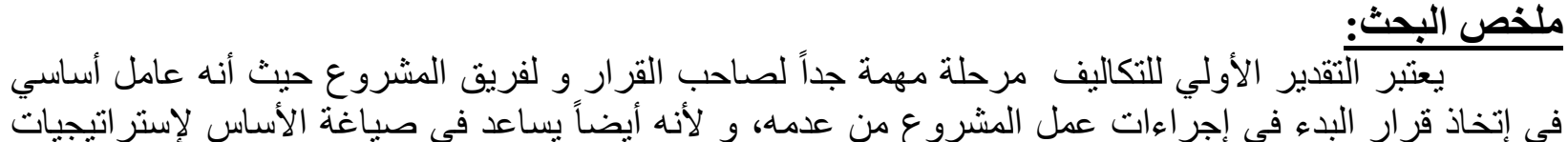

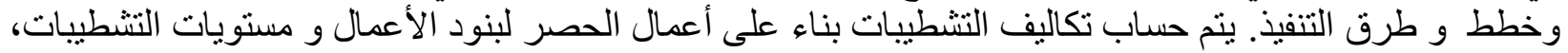
و تختلف مستو يات التشطيبات بناء على مو اصفات بنود الأعمال و نوع التوع الخامات المستخدمة.

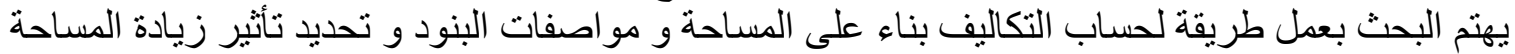

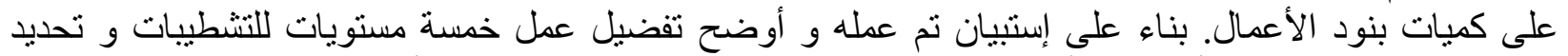

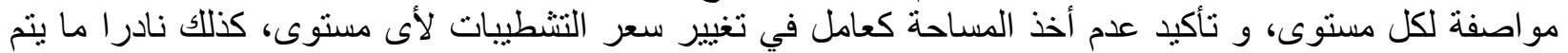

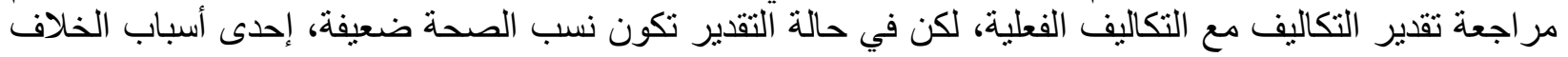
مع العميل الحسابات المالية و بنسبة ليست قلئلة اليلة. فبناء علي ذللك تم حصر جميع بنود الأعمال محل الدراسة لوحدات سكنية (تم تعديل بعض البعات ابعاها لتعطى

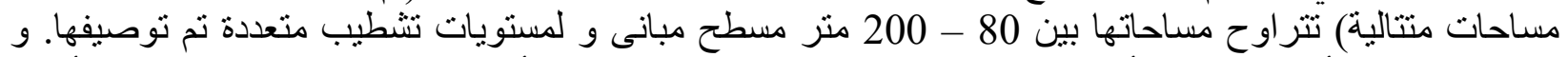

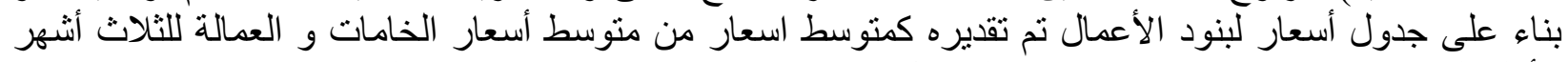

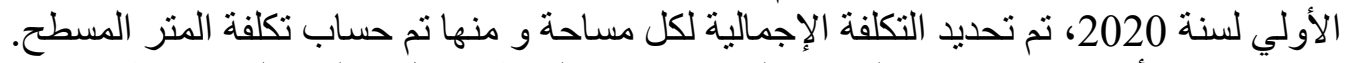

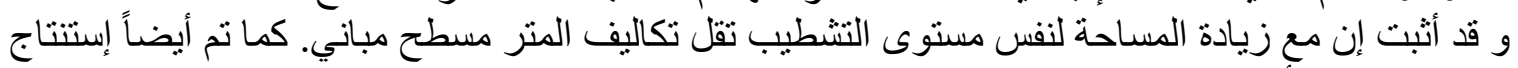
معادلات تعطي إمكانية أكبر لحساب التكاليف، بحيث بمكن بادئ بالمعادلات حساب تكاليف التشطيب لإي مساحة بدون

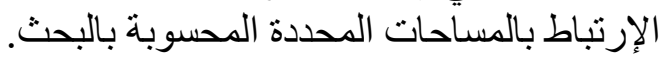
و قد تم تطبيق المعادلة لحساب تكلفة نثطيبات وحدات سكنية، التي أعطت نتائج مقاربة لحسابات التكاليف

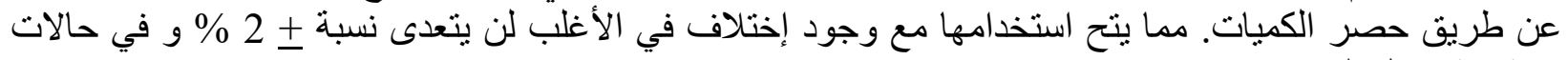
إستثنائية يصل الى بـ 4 \% و توصي الدراسة المهتمين و العاملين بمجال التثطيبات أخذ مساحة المباني في الإعتبار لتخمين تكاليف التشطيبات و التي ستعطي تقدير أدق بإستخدام المعادلات المقترحة. الكعار التشطيبات الاستدلية Key words: التكاليف ـ تكاليف التشطيات ـ المساحات السكنية و تكاليفها ـ المباني السكنية ـ

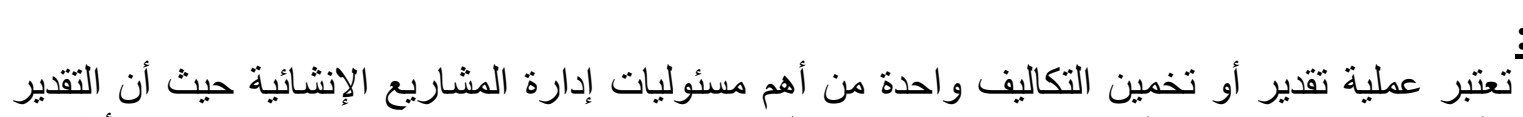

المقدمة:

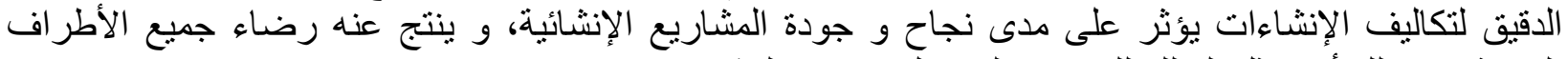

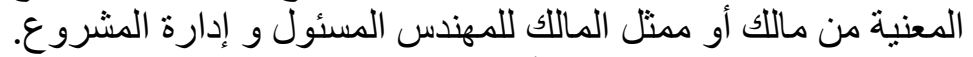

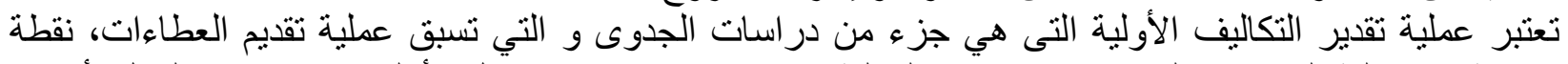

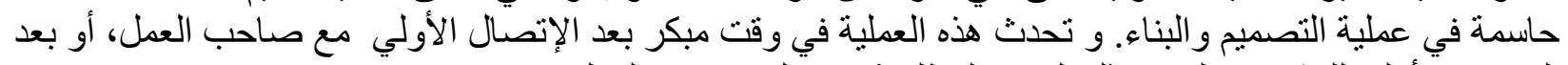

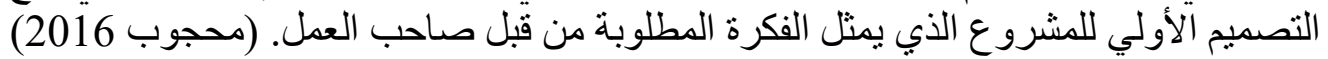

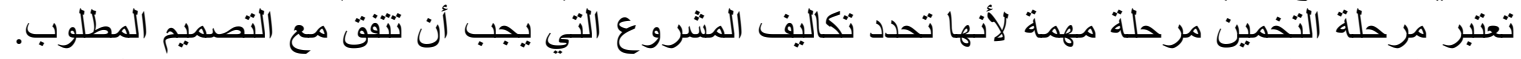
فإذا كانت هذه التكاليف المقدرة غير صحيحة فإنه يتتج عنه بالتبعية تجاوز الميز النية و الذي يظهر في مر احل لاحقة الني 
من مراحل المشروع. أما المبالغة في تقدير التكاليف بالمرحلة الأولي فإن هذا قد يعني أن المشروع قد لا يتم الإستمر ار فيه. (Raisbeck and Aibinu 2010). يتم عمل حسابات التكلفة ثلاث مرات: - ـ الأولي فى مرحلة دراسات الجدوي، حيث لا يكون متوفر غير دراسات الجدوي الأولية و جدول معماري

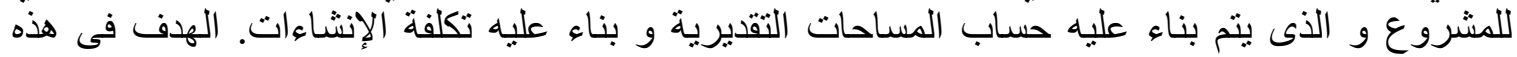

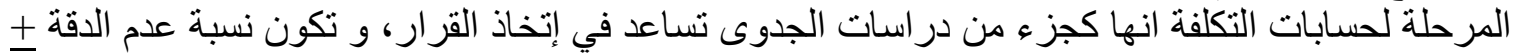
25 \% من التكلفة الفعلية. ـ ـ الثانية بعد الإنتهاء من عمل التصميمات و الرسومات الفنية بناء علي جداول الكميات و المواصفات، و تكون نسبة عدم دقة التقدير + 10 \% م الإن من التكلفة الفعلية. - ـ الثالثة بعد الإنتهاء من إتمام بناء المشروع و ت تكون في هذه المرحلة بيانات الحسابات دقيقة لحسابات التكاليف

الفعلية للمشروع. (Helmus 2002)

إحدى طرق تقدير التكاليف طريقة وحدة المساحة أو الحجم، يتم إستخدام هذه الطريقة في مرحلة دراسات

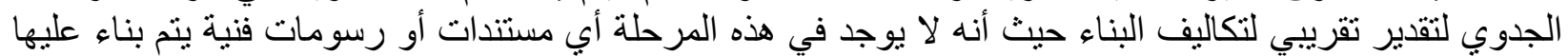

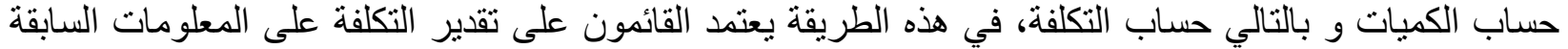

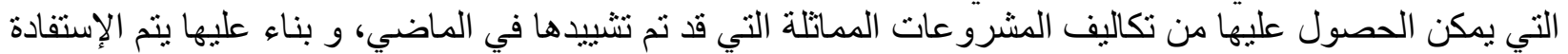

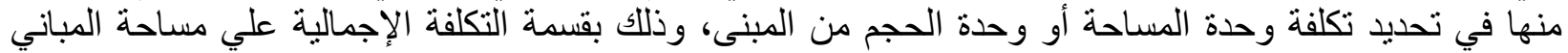

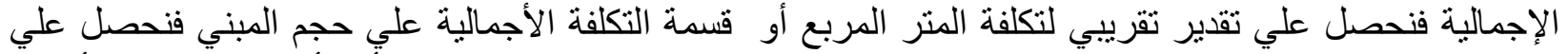

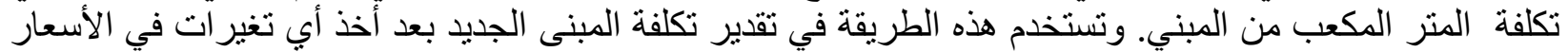

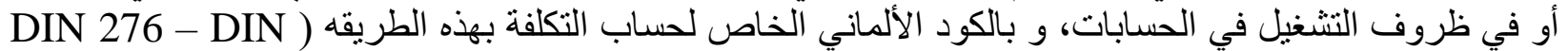

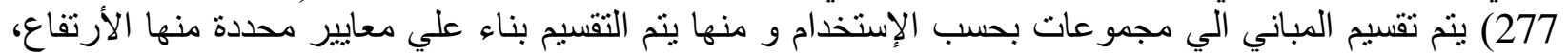
مبانى متصلة أو منفصلة، مو اصفات محددة، و بناء على ثلاث مستويات من التنتطيبات يتم تحديد التكاليف المقدرة و

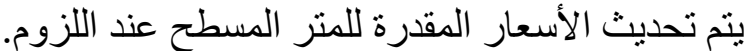

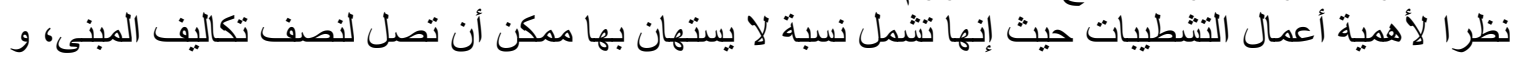

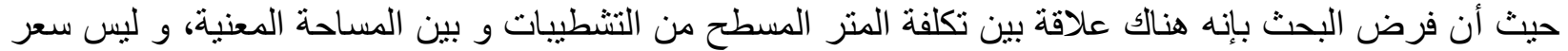

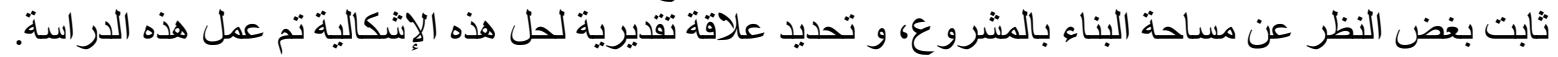

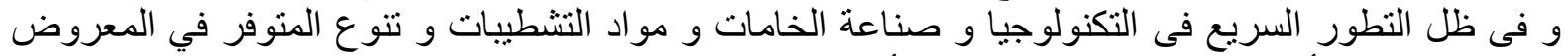

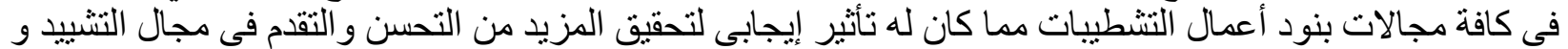

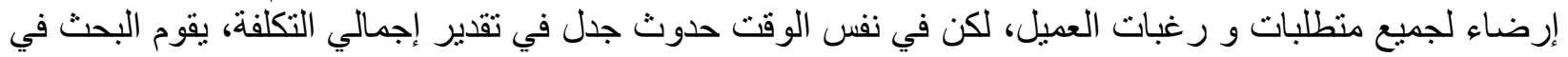

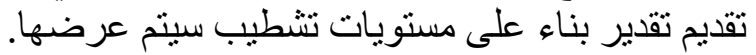

قد تتاول البحث در اسة حساب التكاليف بناء على مشاريع سابقة مثيلة لتكون سعر الوحدة عبارة عن منوسط

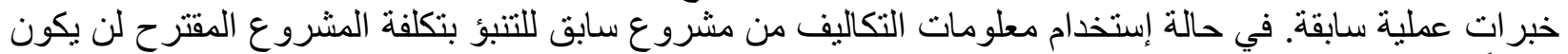

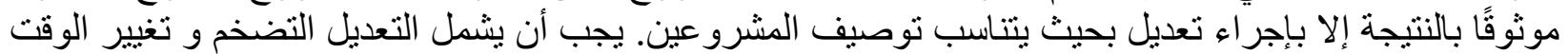

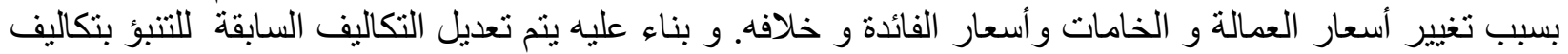

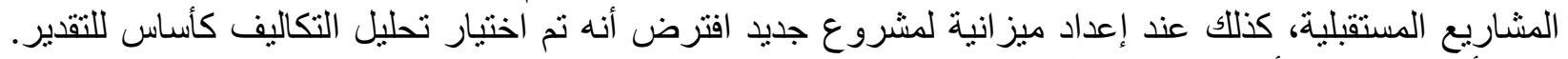

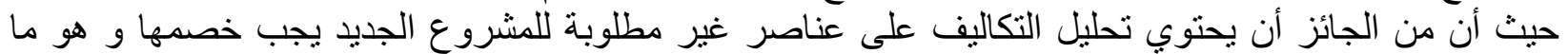

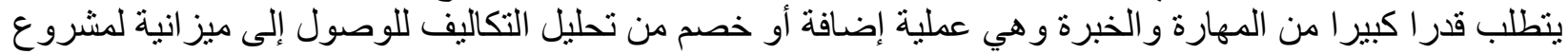

جديد. (Elbeltagi, 2016)

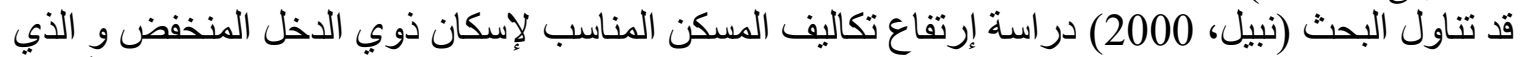

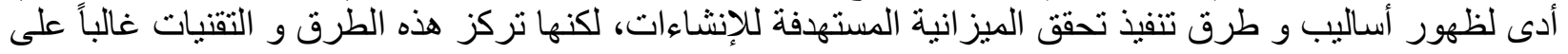


تكاليف البناء الإنشائية المباثرة فقط دون إضافة تكاليف التشطيبات، و قد قام بطر ح بعض المقترحات الفنية لتخفيض

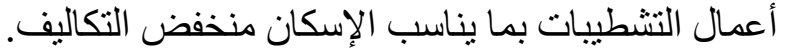

كما قام (نبيل، 2000) بعرض تفصيلي لأقسام عناصر إنتاج المسكن لتشمل على عنصرين أساسيين:

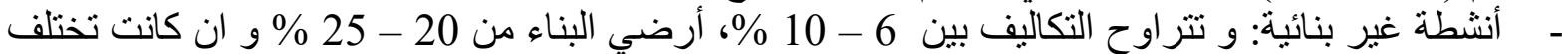

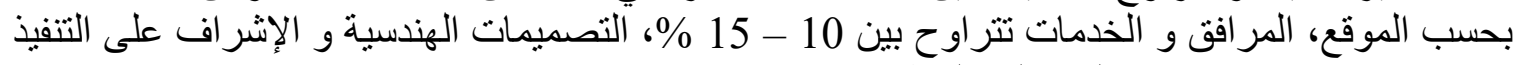

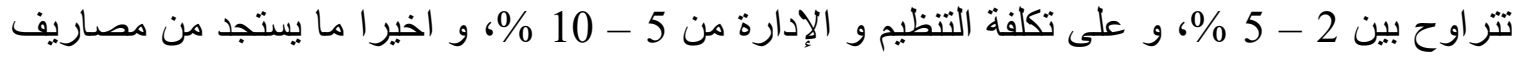
غير منظورة تتر اوح بين 2 - 10 \% عال

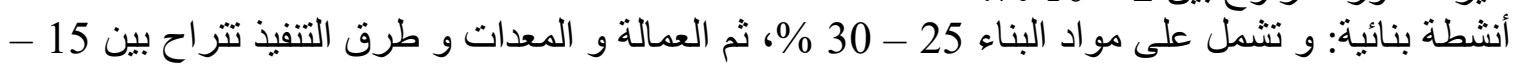
$\% 20$ و يلاحظ عدم ثبات النسب حيث أنها تختلف بناء على كثير من العو امل منها الموقع و مستوى التشطيب. وقام

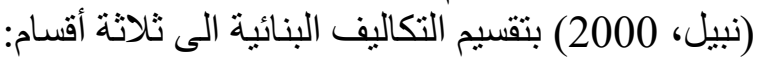
- - الأساسات و هو كل ما أسفل سطح الأرض، و تشنمل على نسبة حو الي 10 \%

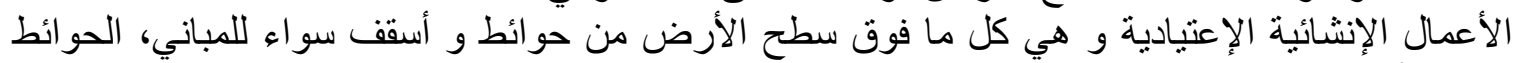

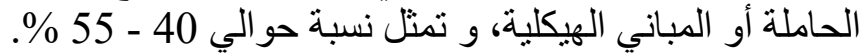

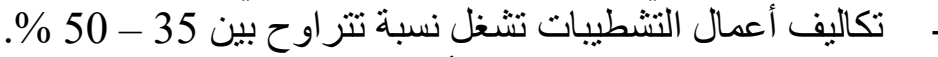

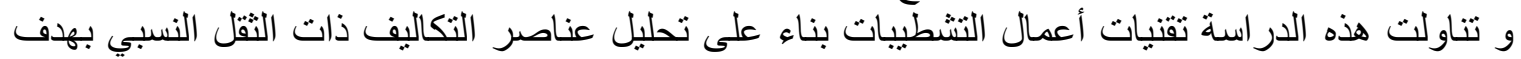

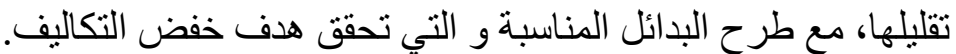

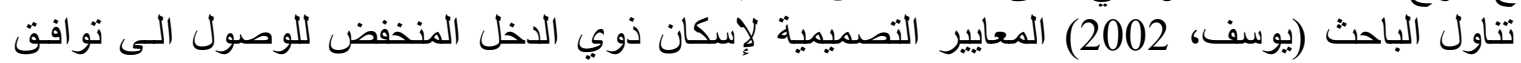

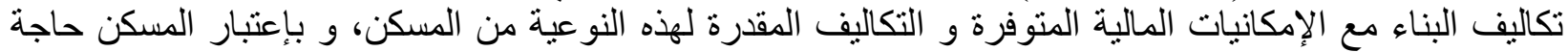

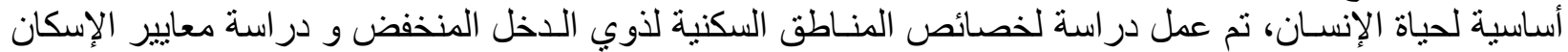

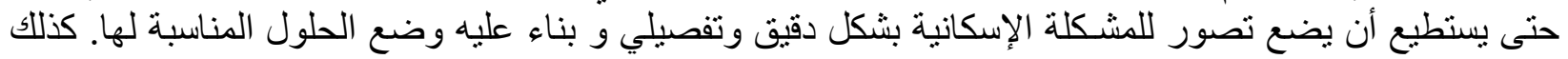

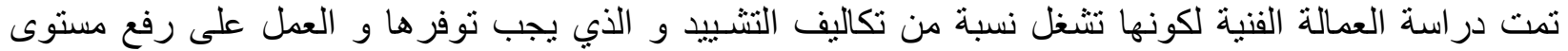

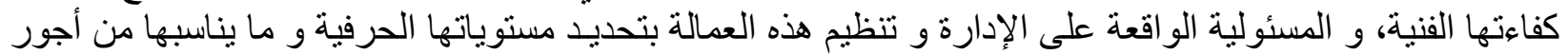

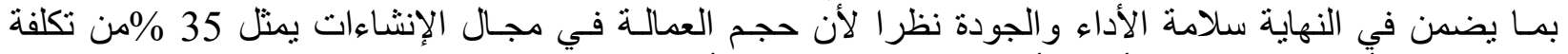

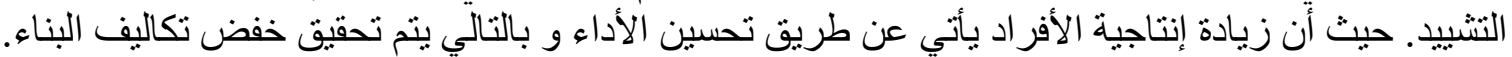

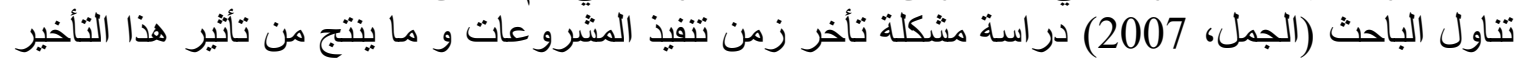

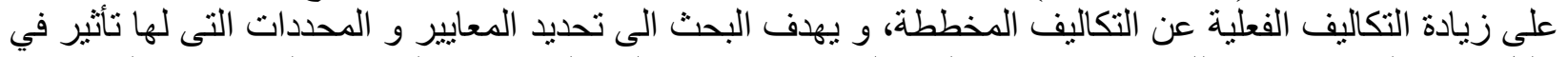

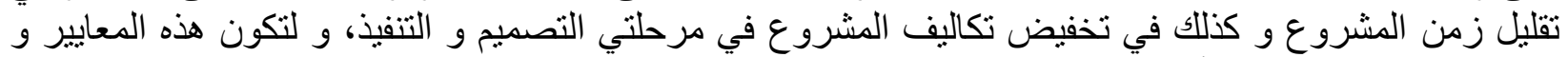

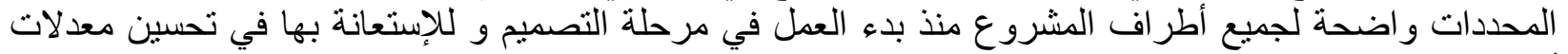

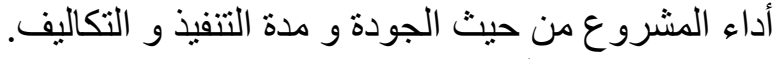

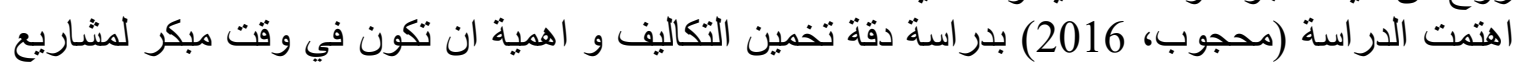

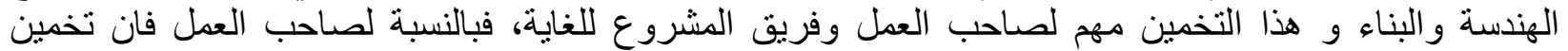

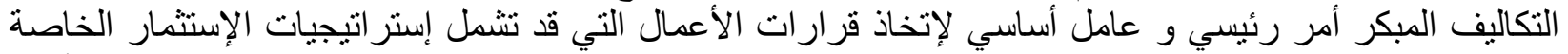

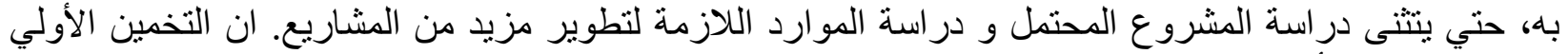

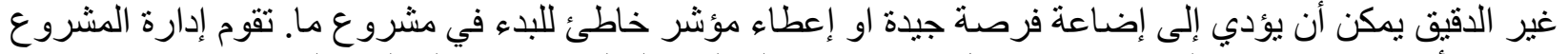

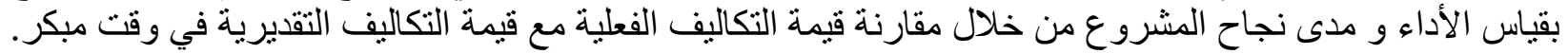

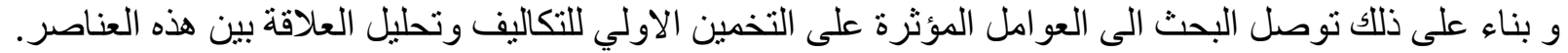

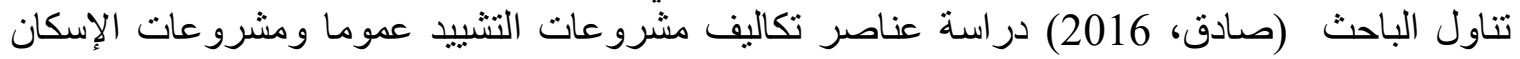

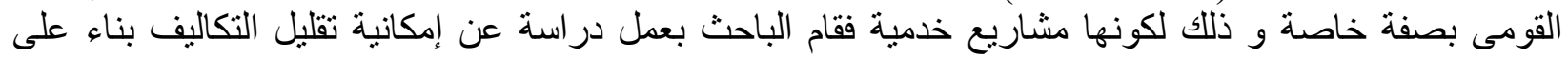

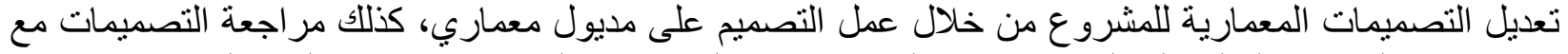

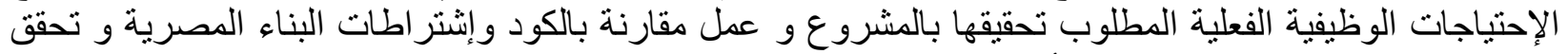

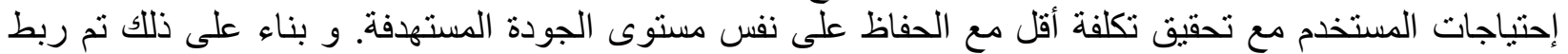
مرحلة التصميم و مرحلة إعداد المو اصفات الفنية بالتكاليف وذللك لحذف أبي تكاليف غير ضرودة الفية. 
تناول الباحث (عبد الغني، 2017) مشكلة زيادة تكاليف بعض المشاريع الإنشائية مما لها من أهمية كبيرة،

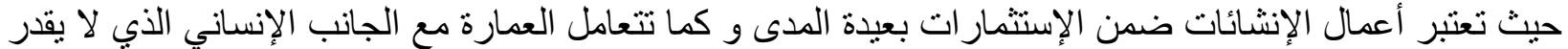

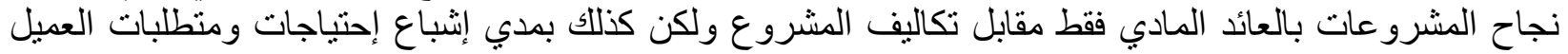
وهو ما تناوله الباحث من خلال منهج عملي، التكاليف لها دور مهم في جميع مراحل المشروع، و تناولت لت دراسة

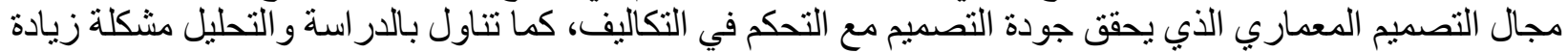

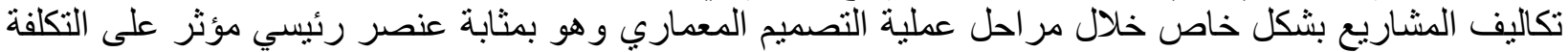
الإجمالية للمشروع. وقد توصل للعو امل التى تؤثر على التكاليف خلال مر احل التصميم المعماري و التي تحكمها

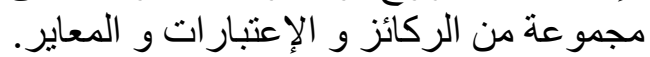

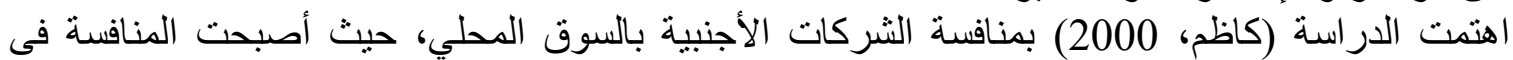

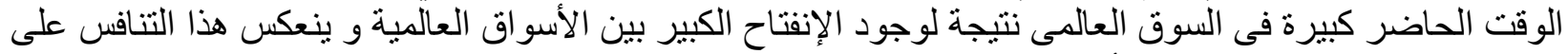

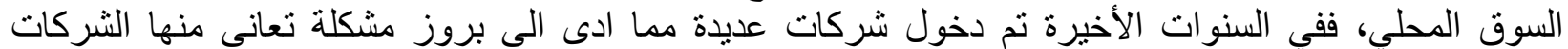
المحلية، هدفت الدارسة الى بناء إطار نظرى عن هندسة القيمة لتوضيح مفهومها وأهدافها وفو ائدها و عناصر هائ و

كيفية تطبيقها.

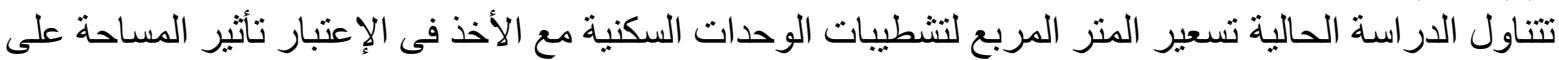

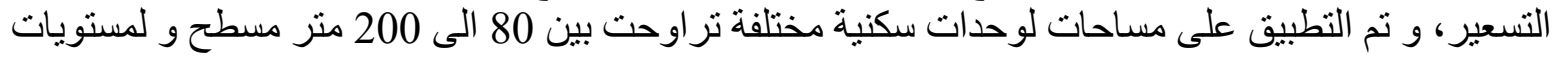
تشطيب متعددة.

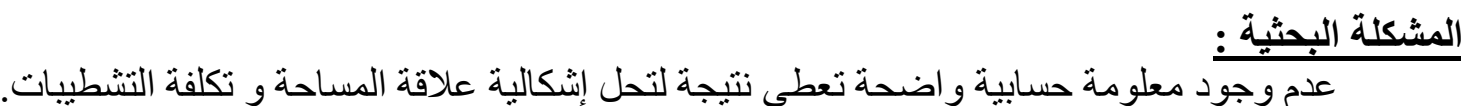
اهداف البحث: 1. تحديد مستويات للتشطيبات لتكون دليل للمقارنة لمستوى النشطيب و الأسعار. 2. تسعير المنر المربع لتشطيبات الوحدات السكنية مع الأخذ فى الإعتبار تأثثير المساحة على التسعير.

المنهجية:

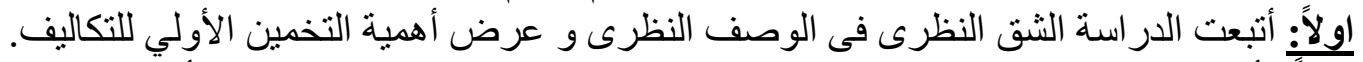

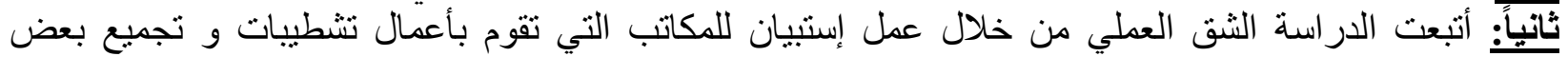

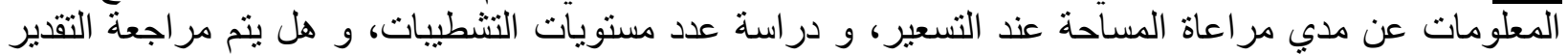

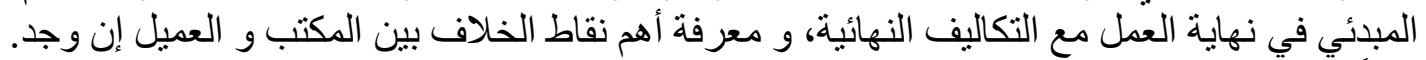

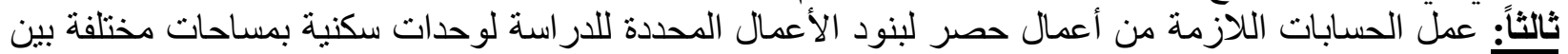
80 اللى 200 متر مسطح، و تثبيت أسعار للمو اصفات المفترضة، لإستتناج علاقة بين المساحة و التكاليف لمستويات التشطيب المختلفة.

تم تصميم إستمارة الإستبيان بهذف جمع معلومات لمعايير محددة لتأكيد او نفي بعض النقاط المهمة للبحث و التى

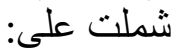
- - عدد مستويات التشطيب التي تقوم بها الثركة،

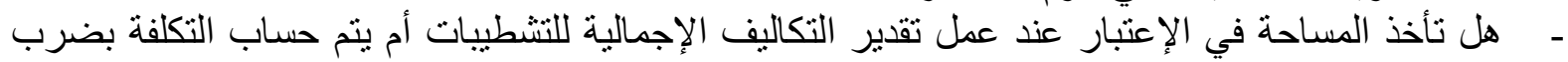

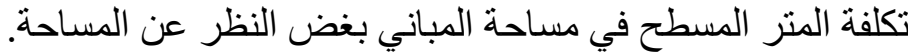
- نسبة صحة تقدير التكلفة، عن طريق مقارنة التكاليف الفعلية بعد الإنتهاء من التنفيذ بالتكاليف المقدرة، و في حالة انه يتم ما هى نسبة صحة التقدير . - كذلك في حالة وجود خلاف مع العميل (المالك) ما هو نوع نهيلة الخلاف (فني، تعاقدي أو مالي). 
تم توزيع الإستبيان لعدد (150) مهندس من مكاتب و شركات مختلفة و إستجاب عدد (132) مهندس بنسبة (88 \%) (25)،

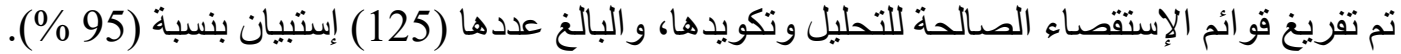

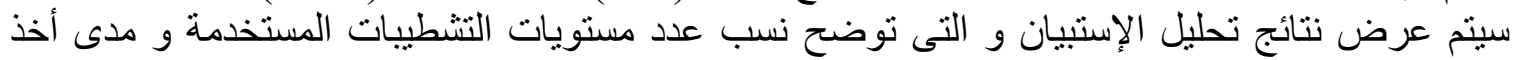
المساحة في الإعتبار عند تقدير التكاليف، كذللك مر اجعة التقدير بعد إنتهاء العمل مع التكاليف الفعلية.

النتائج :

تحليل الإستبيان:

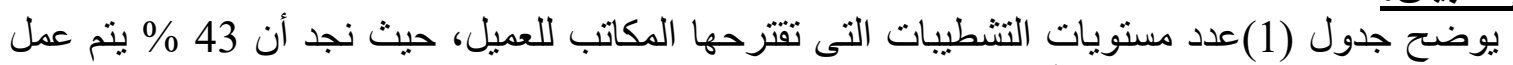

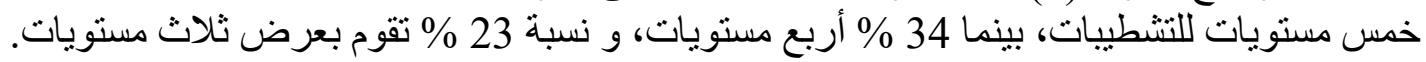

شكل (1) نسب عدد مستو ايات التشطييات

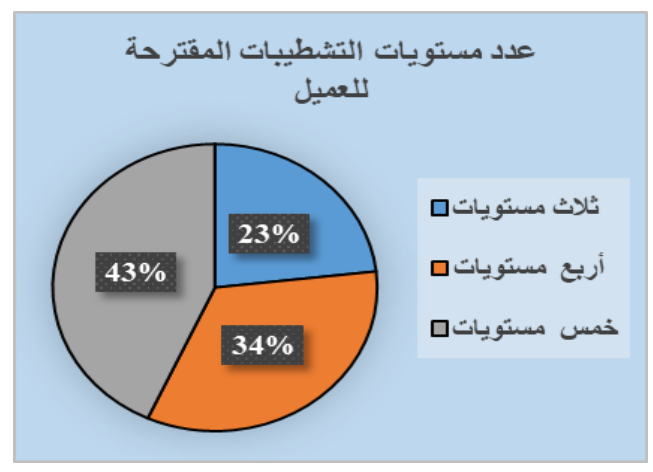

\begin{tabular}{|c|c|c|}
\hline$\%$ & المهندسين & المقترحة مستويات التشيل \\
\hline 23 & 29 & ثلاث مستويات \\
\hline 34 & 42 & أربع مستويات \\
\hline 43 & 54 & خمس مستويات \\
\hline
\end{tabular}

يوضح جدول (2) و شكل (2) عدد الثركات التى يأخذ المساحة في الإعتبار عند تقدير نكاليف التشطيبات شكل (2) نسب تقدير التكاليف على أساس المساحة هى 3 \% و هي نسبة قليلة جدا. أخذ المساحة في الإعتبار عند تقدير

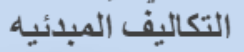

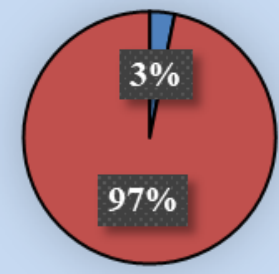

\begin{tabular}{|c|c|c|}
\hline$\%$ & المهندسين & تقدير التكاليف ألمبائية الاعتبار عند \\
\hline 3 & 4 & نعم \\
\hline 97 & 121 & $y$ \\
\hline
\end{tabular}

و يوضح جدول (3) و شكل (3) الثركات التي تقوم بمقارنة التكلفة المقدرة بالتكاليف الفعلية بعد إنتهاء

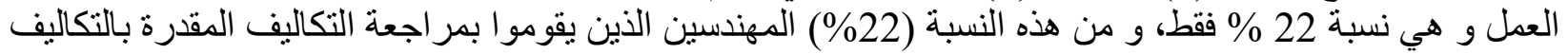
الفعلية بالجدول (4) و شكل (4) تكون نتيجة مدى صحة تقدير التكاليف مع التكاليف الفعلية و التي تعطي نتيجة

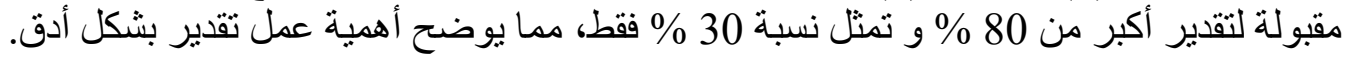


شكل (3) مر اجعة التقدير بعد إنتهاء العمل

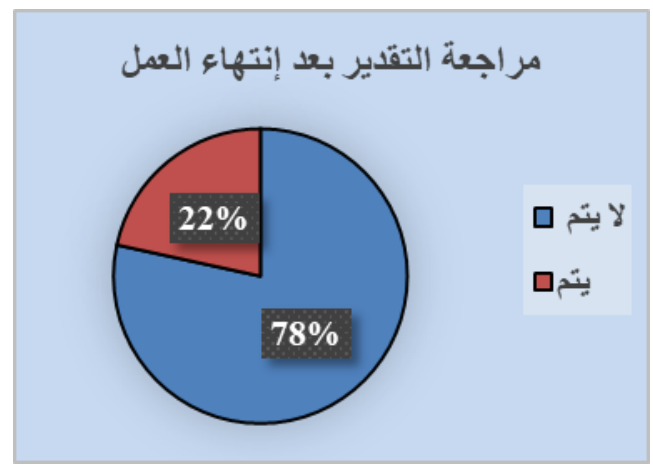

جدول (3) مر اجعة التقدير بعد إنتهاء العمل

\begin{tabular}{|c|c|c|}
\hline$\%$ & المـندسين & العمل مع التكاليف التقدير الفعلية إنتهاء \\
\hline 78 & 98 & لا يتم \\
\hline 22 & 27 & يتم \\
\hline
\end{tabular}

شكل (4) مدى صحة تقدير التكاليف

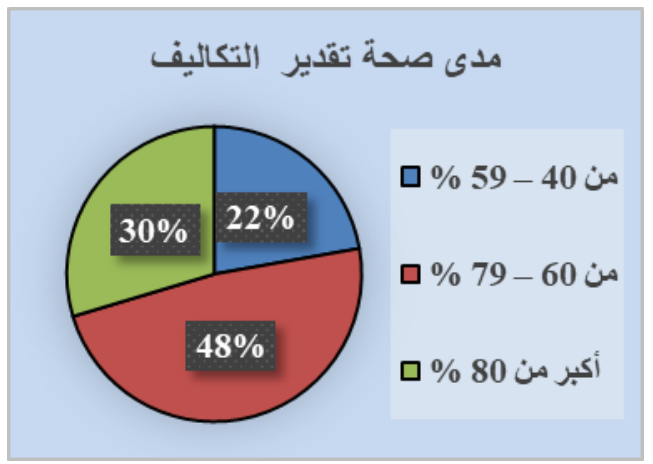

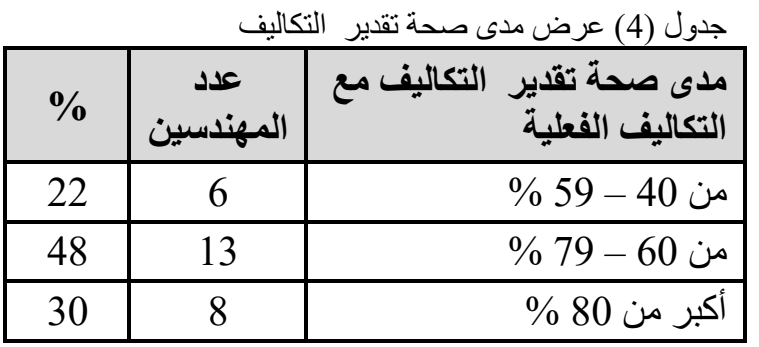

يوضح جدول (5) و شكل (5) نوع الخلاف الذى ينشأ بين الثركة و العميل و يأكد وجود نسبة كبية من بن الخلافات بسبب الحسابات المالية كذلك الناحية الفنية(58\%)، مما يأكد أهمية وجود معايير أدق لتقدير التكاليف.

$$
\text { شكل (5) نوع الخلاف مع العميل }
$$

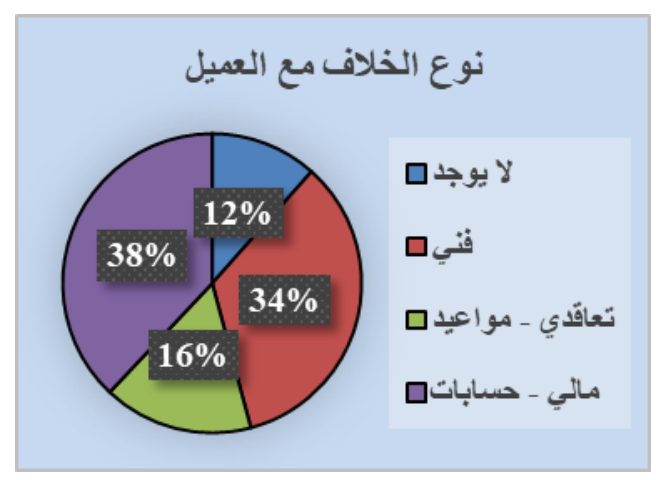

\begin{tabular}{|c|c|c|}
\hline & التكاليف & جدول (5) عرض مدى صحة تقدير \\
\hline$\%$ & المهندسين & نوع الخلاف مع العميل \\
\hline 12 & 25 & لا يوجد \\
\hline 34 & 74 & فني \\
\hline 16 & 35 & تعاقدي - مو اعيد \\
\hline 38 & 82 & مالي - حسابات \\
\hline
\end{tabular}

\section{ملناء على ما تم استخج الاستبيان:}

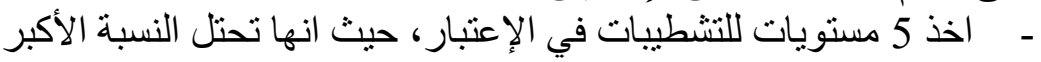

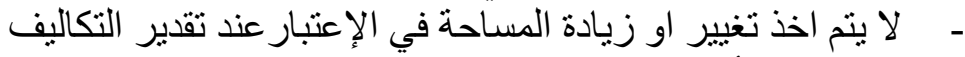
- لا يتم في الأغلب مر اجعة تقدير التكالّف بعد إنتهاء العمل مع التكاليف الفعلية 
- في حالة عمل مقارنة تقدير التكاليف مع التكاليف الفعلية و مدى صحتها كانت نسبة من 60 - 79 \% تقترب من النصف 48\% - الخلافات مع العميل، كانت الخلافات مالية أكبر نسبة و هى 38 \%.

\section{تحديا مستويات للتشطيبات:}

\section{حسابات تكلفة المتر المسطح للتشطيبات:

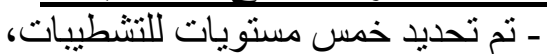
ـ ـ ثم تم تحديد سعر كل بند من بنود الأعمال، عن طريق تقدير الأسعار بمتوسط أسعار الثلاث أثشهر الأولي - و و بناء على مساحات مختلفة للوحدات السكنية تم عمل حصر لبنود الأعمال الرئيسية، بسنة

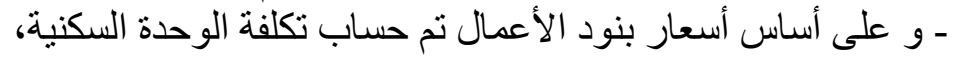
- 2 - و بالتالى حساب تكلفة المتر المربع.

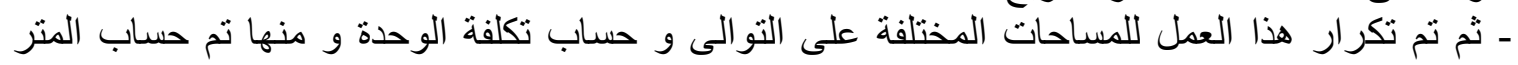
المربع، و بناء على نتائج اسعار المتر المربع للمساحات المختلفة تم تحديد العلاقة بين المساحة و تسعير المتر المربع.

\section{مستويات التشطيب المقترحة:}

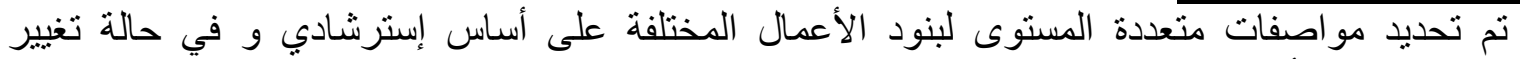
مواصفته و التي يمكن أن تؤدي لتغيير تكلفة الخامات و بالتالي تغيير التكاليف الإجمالية للبند ثم على تكلفة المتر المسطح مباني.

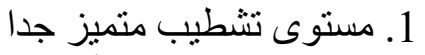

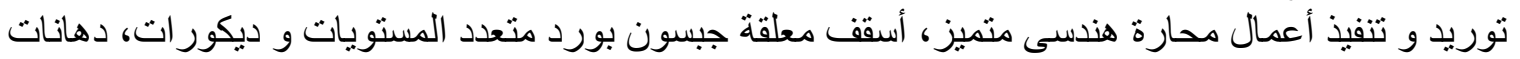

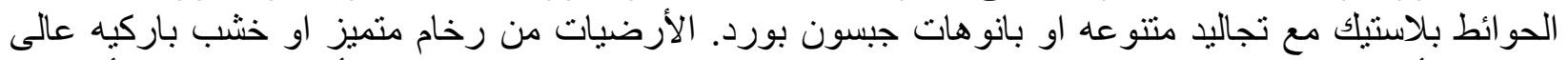

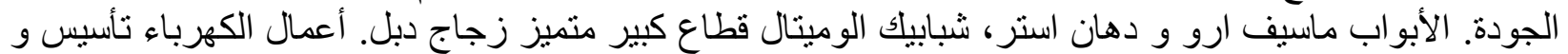

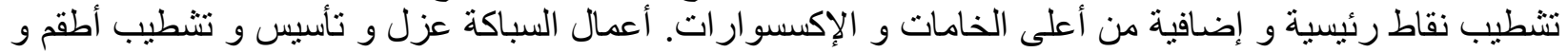

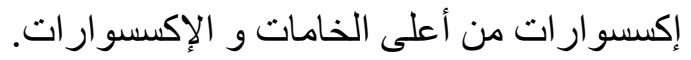

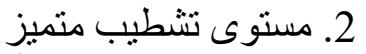

توريد و تنفيذ أعمال محارة هندسى، أسقق معلقة جبسون بورد مستويين مع بيت نورئ نور، دهانات الحوائط

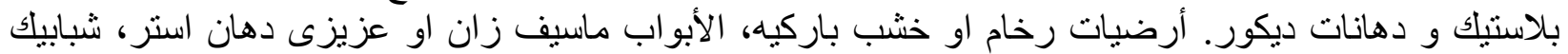

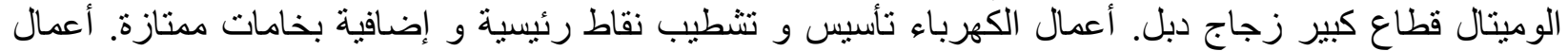

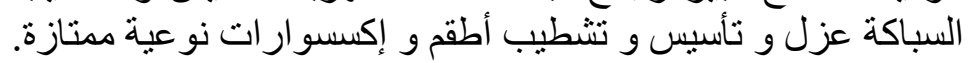

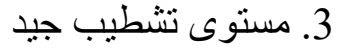
توريد و تنفيذ أعمال محارة، أسقق معلقة جبسون بورد بسيط، الحوائط دهانات بلاستيك و دهانات ديكور

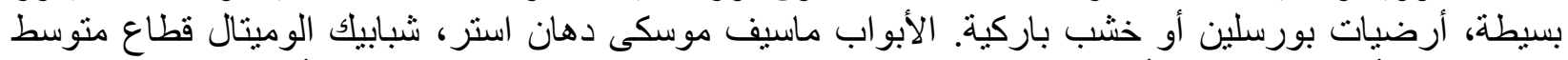

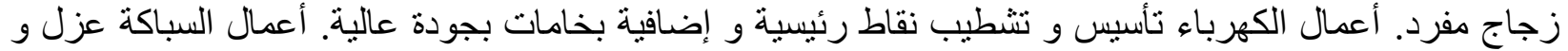

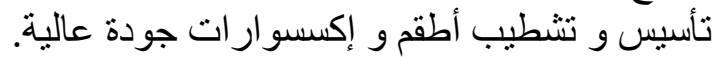

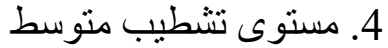

توريد و تنفيذ أعمال محارة بسيطة، دهانات الأسقف مع كر انيش على بياض مصيص، دهيط دهانات الحوائط

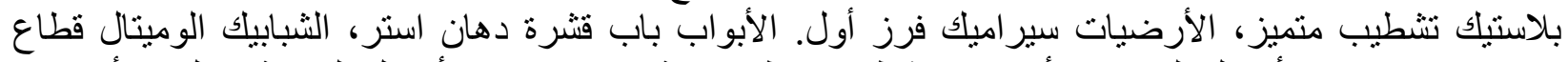

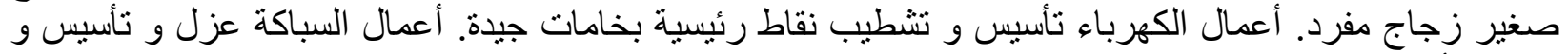

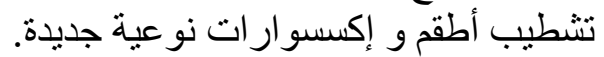

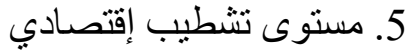




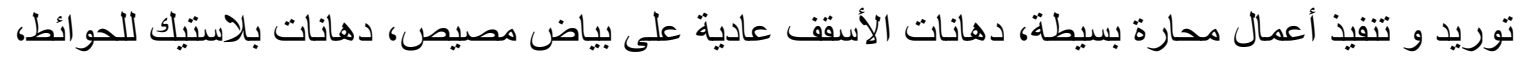

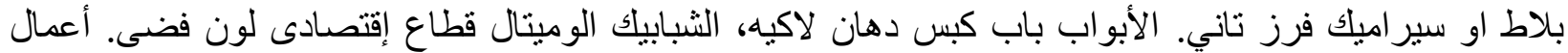
الكهرباء تأسيس و تثطيب ألنقاط الأساسية فقط. أعمال السباكة عزل و تأسيس و تثطيب كامل خامات إقتصادية.

طريقة التسعيز:

أسعار بنود اعمال التشطيب المقترحة:

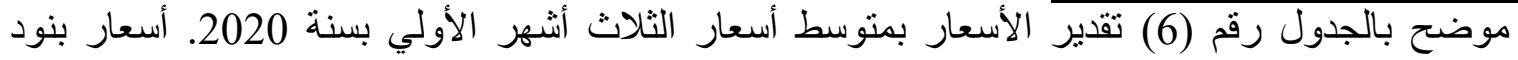

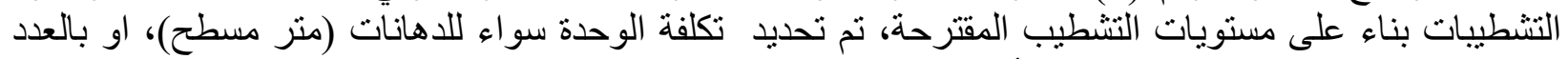

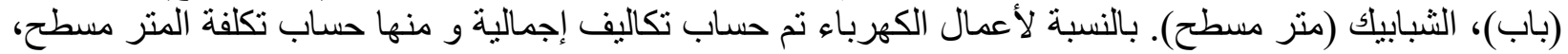

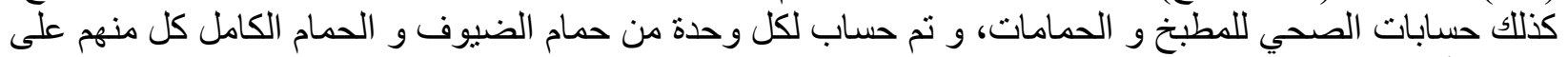
حدة. الأسعار التي بالجدول نتمل جميع التكاليف المبانشرة و الغير مبانشرة و التكاليف الإضافية (سعر المتر

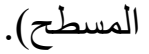

\begin{tabular}{|c|c|c|c|c|c|c|}
\hline \multicolumn{5}{|c|}{ مستوى تثطيب / تكلفه الوحده (جنية) } & \multirow{2}{*}{ 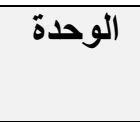 } & \multirow[b]{2}{*}{ بنود الأعمال } \\
\hline 5 & 4 & 3 & 2 & 1 & & \\
\hline 80 & 130 & 170 & 200 & 250 & 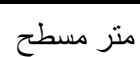 & بياض و دهانات حوائطو أسقف \\
\hline 100 & 120 & 140 & 180 & 220 & متر مسطح & سبر اميك حوائط \\
\hline 130 & 150 & 170 & 200 & 250 & متر مسطح & سبراميك ارضيات \\
\hline 130 & 180 & 240 & 350 & 500 & متر مسطح & ارضيات \\
\hline 800 & 1200 & 2000 & 3000 & 4000 & متر مسطح - متح & شباك /م2 \\
\hline 2000 & 4000 & 6000 & 8000 & 10000 & بالعدد & باب \\
\hline 250 & 280 & 380 & 440 & 500 & مت مسطح & 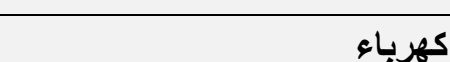 \\
\hline 5000 & 10000 & 20000 & 30000 & 40000 & بالعدد & صحى / مطبخ \\
\hline 10000 & 20000 & 30000 & 50000 & 70000 & بالعدد & صحي / حمام \\
\hline 3000 & 5000 & 8000 & 10000 & 15000 & مالعدد & صحي / حمام ضيوف \\
\hline
\end{tabular}

المساحات التي تم على أساسها حسابات التكاليف:

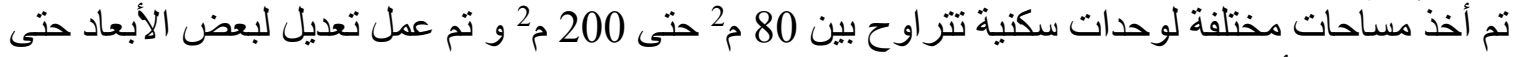

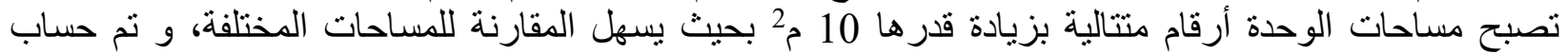

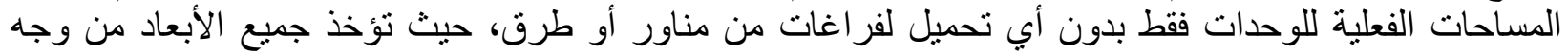
الجدران الخارجية لإحتساب مساحات البناء.

نتائج حسابات التكاليف الإجمالية لتثطيبات المتر المسطح:

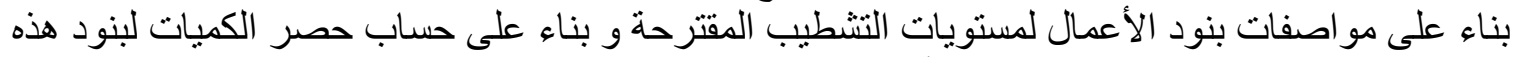

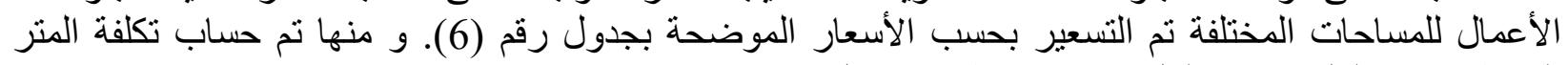
المسطح مباني لكل مساحة لكل مستوى تشطيب بجدول رقم (7). 
مع الأخذ في الإعتبار جميع الوحدات تتكون من 3 غرف و مطبخ و حمام و حمام ضيوف، ماعدا المساحة

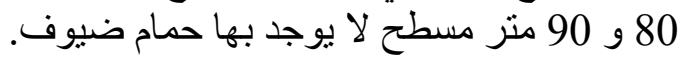

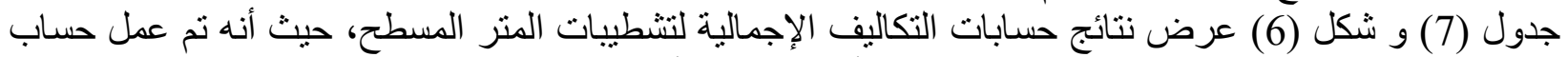

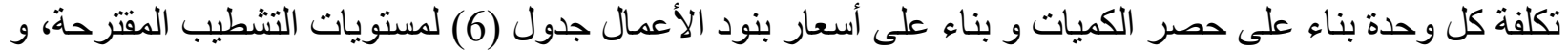
بناء عليه تم حساب التكلفة الإجمالية و منها تم حساب تباء (السعر ) للمتر المسطح.

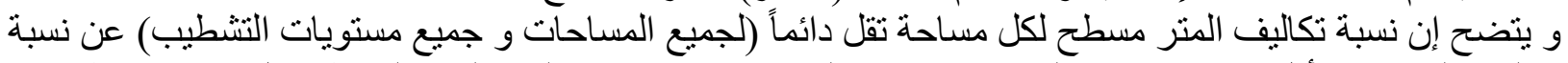

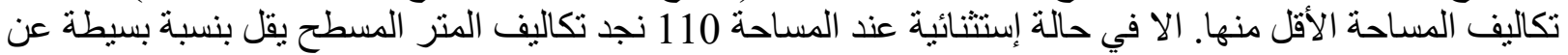

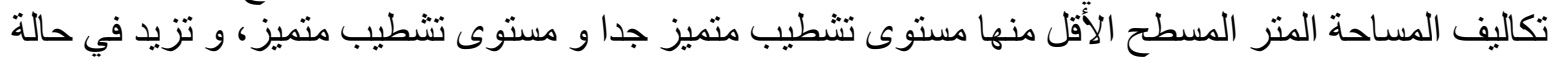

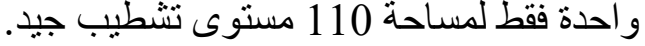
بالجدول (7) نسبة التكلفة للمساحة الأقل = = تكلفة المتز المسطح للمساحة الأقل

و يتضح من الثنكل (6) ان منحنى تكاليف المساحات للمستويات المختلفة مستوي في الإنحدارـ مما يعطي نتيجة نسبة

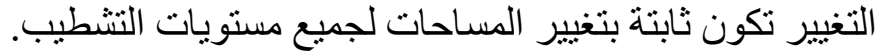
جدول (7) التكاليف الإجمالية للمتر المسطح لكل مستوى تثطيب و نسبة النكلفة للمساحة الأقل

\begin{tabular}{|c|c|c|c|c|c|c|c|c|c|c|}
\hline \multicolumn{11}{|c|}{ السعر (جنية / متر مسطح) } \\
\hline اللتكفة اللمساحة & تقتطيب & اللألتسلفة & متثطيب مستو & اللالكسلفة & تثنطيب & اللألمسلفة & متشطيب & اللمسبة & متثطيب متميز & $2 p$ \\
\hline & 1240 & & 1923 & & 2816 & & 3869 & & 5021 & 80 \\
\hline 94 & 1166 & 93 & 1795 & 93 & 2615 & 92 & 3575 & 92 & 4633 & 90 \\
\hline 99 & 1149 & 97 & 1739 & 95 & 2481 & 94 & 3376 & 95 & 4379 & 100 \\
\hline 99 & 1132 & 99 & 1729 & 101 & 2504 & 100 & 3384 & 100 & 4389 & 110 \\
\hline 96 & 1090 & 96 & 1655 & 95 & 2386 & 95 & 3214 & 95 & 4162 & 120 \\
\hline 96 & 1047 & 96 & 1582 & 95 & 2275 & 95 & 3057 & 95 & 3956 & 130 \\
\hline 97 & 1013 & 96 & 1522 & 96 & 2183 & 96 & 2925 & 96 & 3782 & 140 \\
\hline 97 & 985 & 97 & 1474 & 97 & 2108 & 96 & 2816 & 96 & 3637 & 150 \\
\hline 98 & 961 & 97 & 1431 & 97 & 2041 & 97 & 2719 & 96 & 3508 & 160 \\
\hline 98 & 939 & 97 & 1391 & 97 & 1979 & 97 & 2630 & 97 & 3390 & 170 \\
\hline 98 & 916 & 97 & 1353 & 97 & 1921 & 97 & 2549 & 97 & 3284 & 180 \\
\hline 98 & 894 & 97 & 1317 & 97 & 1867 & 97 & 2473 & 97 & 3184 & 190 \\
\hline 99 & 882 & 98 & 1290 & 98 & 1823 & 97 & 2409 & 97 & 3097 & 200 \\
\hline
\end{tabular}




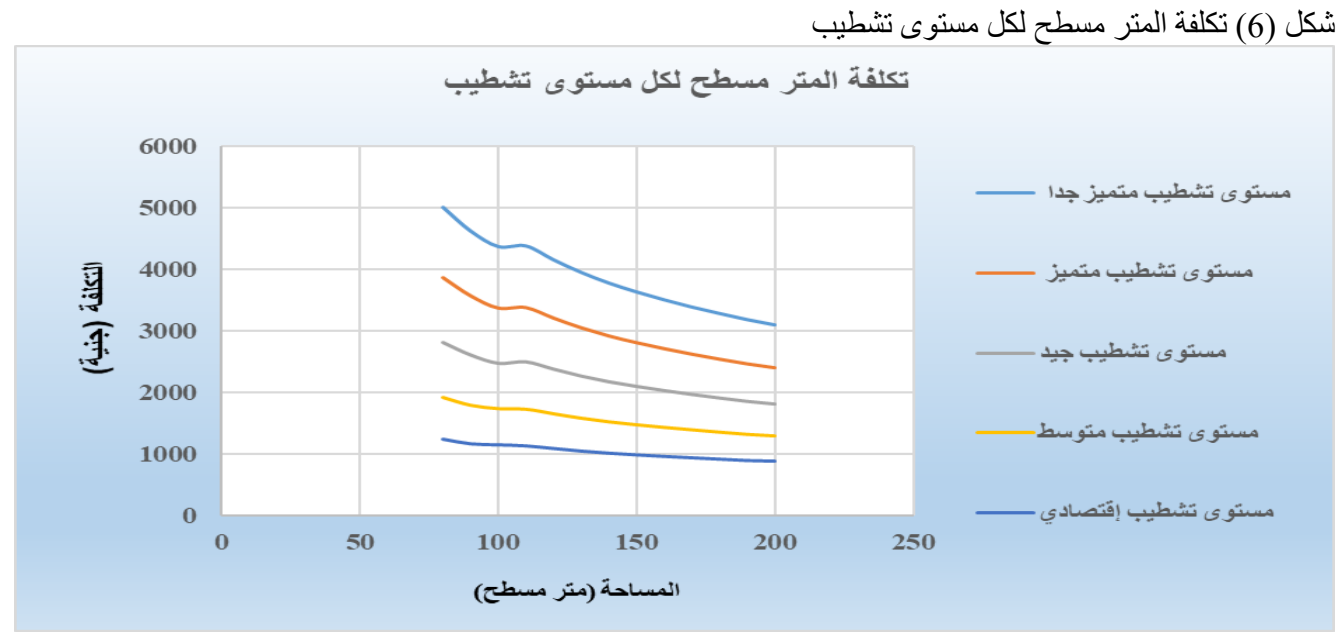

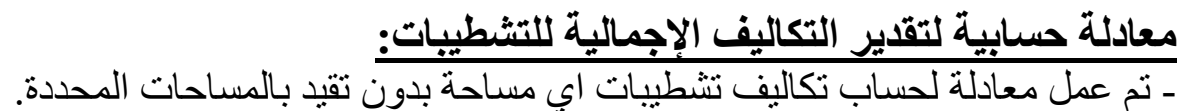

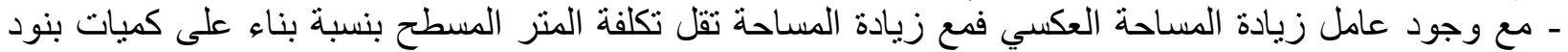
الأعمال و هذه النسبة تم إدخالها في المعادلة بين 0.05 - 0.07 حسب مستب فئوى التشطيب، و تم حسابها من الجدول منوسط معامل التغيير من مساحة لمساحة.

- بناء على المعادلة التالية يتم حساب تكاليف الوحدة مستوى تشطيب متميز جا و مستوى تشطيب متميز و مستوى

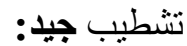
تكلفة الثقة ـ المعادلة التالية لحساب تكاليف الوحدة مستوى تشطيب متوسط: ت تكلفة الشقة $\left.=0.06 \times\left(\frac{80-\mathbf{X}}{10}\right)\right] \times \mathrm{Y} \times 80=\mathrm{d}$ - بالمعادلة التالية يتم حساب تكاليف الوحدة مستوى تشطيب إقتصادي: تكلقة الشقة $\left.=0.07 \times\left(\frac{80-\mathbf{X}}{10}\right)\right] \times Y \times 80=\mathbf{~}=$ 10

حيث أن: Y : تكلفة المتر مسطح لمستوى التشطيب لمساحة 80 متر مسطح (بالجدول رقم 7)

- هدف وجود معادله لحساب تكاليف تشطيبات اي مساحة بحيث تعطي إمكانية أكبر لحساب تكاليف أي مساحة بدون

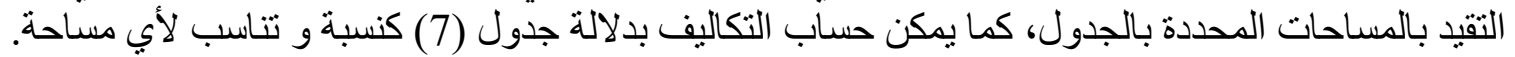
- و يمكن حساب تكاليف التشطيبات باستخدام المعادلة التالية، مع الأخذ في الإعتبار يتم إحتساب التكاليف بدلالة

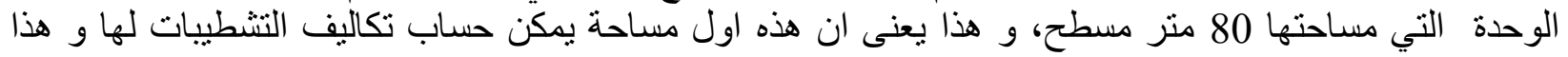

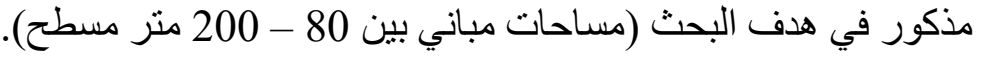




\section{تطييق المعادلة لحساب تكلفة تثطيبات الوحدات السكنية لمستوى تثطيب متميز جدا للمساحة 180:}

$$
\begin{aligned}
& =\left[1+0.05 \times\left(\frac{80-180}{10}\right)\right] \times 5021 \times 80=\underline{180} \\
& 602520=1.50 \times 401680= \\
& \text { تكلفة المنر مسطح = } 602520 \text { × } 180 \text { = } 3347 \text { جنية }
\end{aligned}
$$

فبنطبيق المعادلة معدل التغيير قدره + 1.9 \% مقارتاً بأسعار الجدول (7)، حيث ان تكلفة المتر مسطح للمساحة 180 هب بالجدول هي 3284 جنية/للمتر المسطح.

جدول (8) يوضح نطبيق المعادلة لحساب تكلفة تثطيبات الوحدات السكنية حيث نم تطبيق المعادلة لثلاث مساحات

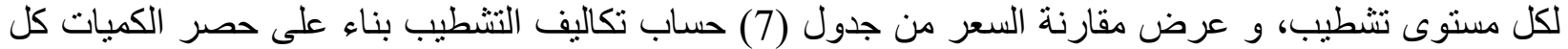
وحدة و السعر بناء على المعادلة و مقدار التغير. و تعتبر نسبة التغير مقبولة كتخمين أولي بناء على المساحة و ولى مستوى التشطيب و أسعار الخامات. مع الأخذ في الإعتبار جميع الوحدات تتكون من 3 غرف و مطبخ و حمام و حمام ضيوف، ماعدا المساحة

\begin{tabular}{|c|c|c|c|c|}
\hline \multirow{2}{*}{$\begin{array}{c}\text { مقار التغيير } \\
\text { \% }\end{array}$} & \multicolumn{2}{|c|}{ السعر (جنية / متر مسطح) } & \multirow{2}{*}{ المساحة / متر } & \multirow{2}{*}{ مستوى التشطيب } \\
\hline & بناء على حصر الكميات & بناء على تطبيق المعادلة & & \\
\hline $0.9+$ & 4379 & 4418 & 100 & \multirow{3}{*}{ مستوى تثطيب } \\
\hline $1.4-$ & 3782 & 3729 & 140 & \\
\hline $1.9+$ & 3284 & 3347 & 180 & \\
\hline $0.9+$ & 3376 & 3405 & 100 & \multirow{3}{*}{ مستوى تثطيب } \\
\hline $1.8-$ & 2925 & 2874 & 140 & \\
\hline $1.1+$ & 2549 & 2579 & 180 & \\
\hline 0.1 - & 2481 & 2478 & 100 & \multirow[t]{3}{*}{ مستوى تشطيب جيذ } \\
\hline $4.3-$ & 2183 & 2092 & 140 & \\
\hline $2.3-$ & 1921 & 1877 & 180 & \\
\hline $0.9-$ & 1739 & 1723 & 100 & \multirow{3}{*}{ مستوى تثطيب } \\
\hline $1.9-$ & 1522 & 1494 & 140 & \\
\hline $1.0+$ & 1353 & 1367 & 180 & \\
\hline $1.6-$ & 1149 & 1131 & 100 & \multirow{3}{*}{ مستوى تثطيب } \\
\hline 0.6 - & 1013 & 1006 & 140 & \\
\hline $2.2+$ & 916 & 937 & 180 & \\
\hline
\end{tabular}

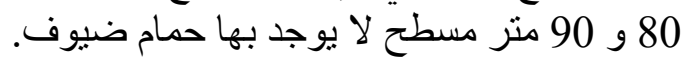

جدول (8) مقارنة التكاليف للمتر المسطح نتيجة أعمال الحصر جدول (7) بالتكاليف نتيجة المعادلة و نسبة التغيير

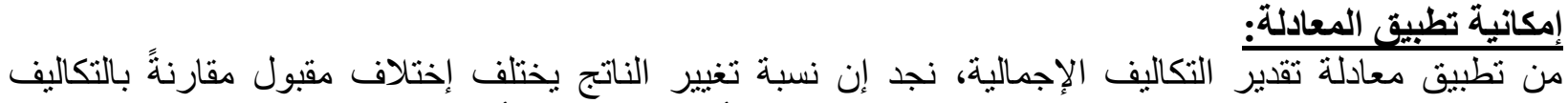

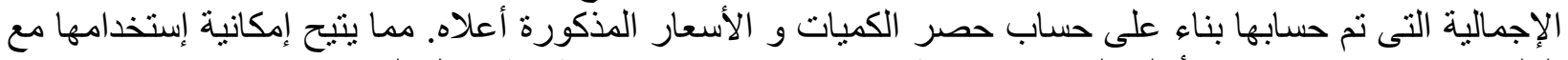
العلم ان هذا الإختلاف في الأغلب لن يتعدى نسبة +2 2 \% و في حالات إستثنائية يصل الى ــ 4 \%

في حالة وجود حمام اخر ، أو حمام ضيوف اخر ينم إضافة تكلفة الوحده كما مبين بالجدول رقم (9)، و هذة الأسعار تم حسابها شاملة جميع التكاليف بما يتناسب مع نو عية التشطيب. 
جدول (9) تكلفة وحدات الحمام و حمام الضيوف الإضافية

\begin{tabular}{|c|c|c|c|c|c|}
\hline تشتطيب & متثطيب متوسط & تشطيب مستوى & متشطيب متيز & 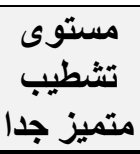 & \\
\hline 19300 & 33300 & 47600 & 73000 & 98800 & الحمام \\
\hline 7650 & 11650 & 16800 & 21500 & 29400 & ح ضيوف \\
\hline
\end{tabular}

كذلك في حالة وجود عدد أكبر من الغرف المفترضة بالبحث، لكل غرفة زئل معامل 1.025، و هذة النسبة المقدرة محسوبة من منوسطات زيادة كميات بنود الأعمال مع زيادة وجود غرفة غرفة لنفس لنس المساحة.

محصلة النتائج و التوصيات: على كميات بنود الأعمال. فبناء علي ذللك تم حصر لجميع بنود الأعمال محل الدر اسة لوحدات سكنية (تم تعديل بعض ابعاها لتعطى

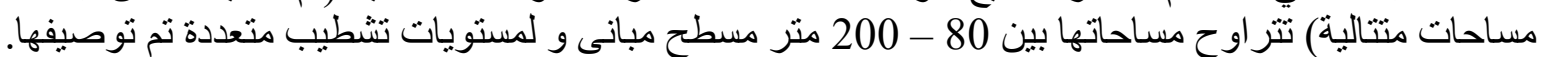

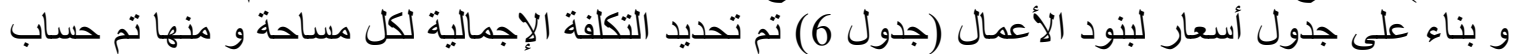

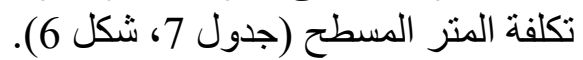
و بناء عليه تم إستتتاج معادلات كمثنال تكميلي بعطي إمكانية أكبر لحساب تكاليف التشطيب، بحيث بمكن

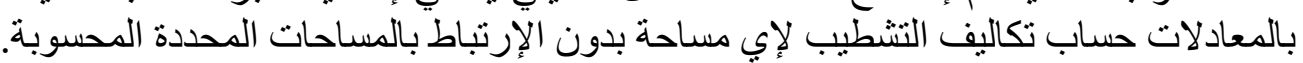

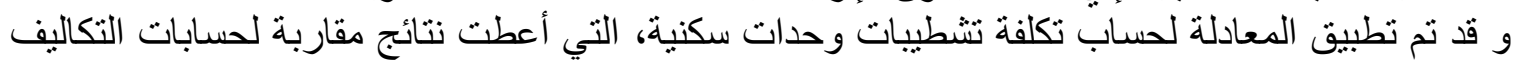

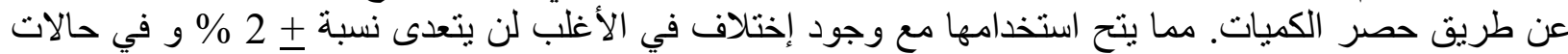

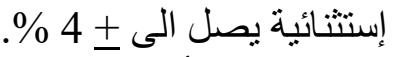
و قد أثبتت الدر اسة إن مع زيادة المساحة لنفس مستوى التشطيب تقل تكاليف المتر مسطح مباني.

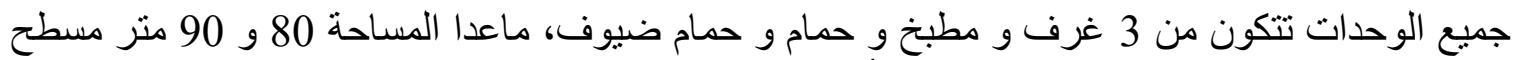
لا يوجد بها حمام ضيوف، في حالة وجود حمام اخر، أو حمام ضيوف اخد ونر يتم إضافة تكلفة الوحده الى التكلفة الكلية للوحدة السكنية. و توصي الدر اسة المهتمين و العاملين بمجال التشطيبات أخذ مساحة المباني في الإعتبار لتخمين تكاليف التشطيبات و التي ستعطي تقدير أدق بإستخدام المعادلات المقات المقترحة.

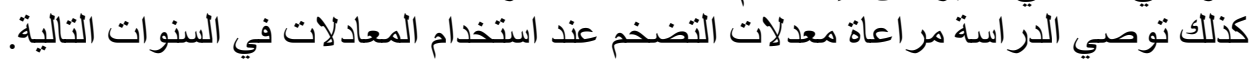

المراجع باجي: اللغة العربية: 1. إبر اهيم عبد الرشيد نصير ، إدارة مشروعات التشييد، دار النشر للجامعات 2006.

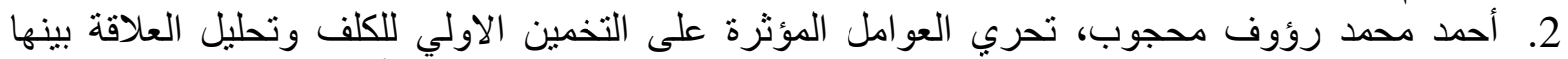

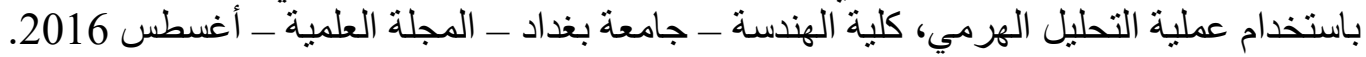

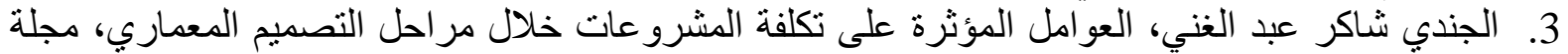

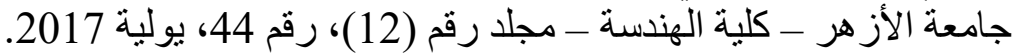

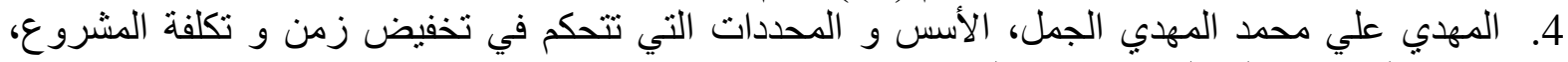
جامعة القاهرة ـ كلية الهندسة - رسالة ماجستير، 2007. 
5. حاتم كريم كاظم، دور هندسة القيمة في تخفيض التكاليف وتطوير المنتجات، رسالة ماجستير غير منشورة، كلية إإقتصاد و المحاسبة ــ العر اق، الكوفة دونة 2000.

6. خالد إبار هيم نبيل، تقنيات التشطيب لإسكان ذوى أدنى الدخول، جامعة حلوان - كلية الهندسة، المجلة العلمية، 2000.

7. محمد عبد الله الريدي، الإدارة الاقتصادية للمشروعات الهندسية، دار الكتب العلمية للنشر و التوزيع 2006.

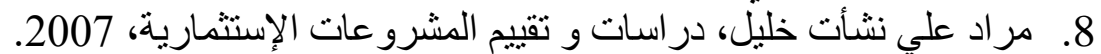

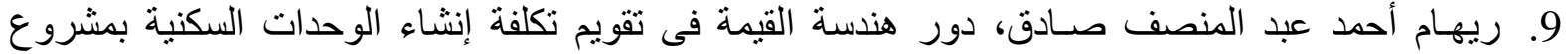

الإسكان القومى، جامعة عين شمس - كلية الهندسة ـ قسم الهندسة المعمارية - رسالة ماجستير 2016.

10. ر رائد محمد صالح يوسف، المعايير التصميمية لاسكان ذوي الدخل المنخفض، جامعة النجاح الوطنية، رسالة

ماجستير،

11. Bhattad Parag R. and Jain R. K. 2015) “An Assessment of Factors Affecting the Accuracy of an Early Cost Estimate for Repetitive Projects" International Journal of Science and Research, Volume 4 Issue 4, April.

12. Emad Elbeltagi Lecture Notes on Construction Cost Estimating, Mansoura University, Mansoura, Egypt, (2016).

13. Gunner, J. and Skitmore, M. (1999) "Comparative analysis of pre-bid forecasting of building prices based on Singapore data ". Construction Management and Economics 17, 635- 646.

14. Ling, Y. Y. and Boo, J. H. S. (2001) "Improving the accuracy of approximate estimates of building estimates". Building Research and Information, 29(4), 312-318.

15. Manfred Helmus, Studium Handbuch für Baumanagement, Universität Wuppertal 2002.

16. Manfred Helmus, Maged Moneer Gad, Honorar-Handbuch für Architekten und Ingenieure, Ernst \& Sohn2002.

17. Oberlender Garold D. and Steven M. Trost, (2001) "PREDICTING ACCURACY OF EARLY COST ESTIMATES BASED ON ESTIMATE QUALITY" Journal of Construction Engineering and Management, Vol. 127, No. 3, May/June, ASCE.

18. Oberlender Garold D. (2000) "PROJECT MANAGEMENT FOR ENGINEERING AND CONSTRUCTION" McGraw -Hill Higher Education, second Edition.

19. Raisbeck, P and Aibinu, A A (2010) "Early stage cost estimation and the relationship of architects to quantity surveyors". In: Egbu, C. (Ed) Procs 26th Annual ARCOM Conference, 6-8 September 2010, Leeds, UK, Association of Researchers in Construction Management, 53-61.

20. Serpell, A. F. (2005) "Improving conceptual cost estimating performance". AACE International Transactions EST.13: EST.13.1-13.6.

21. Skitmore, R. M. and Picken, D. (2000) "The accuracy of pre-tender building price forecasts: an analysis of USA data". Australian Institute of Quantity Surveyors Refereed Journal, 4(1), 33 -39. 
Dr. Maged Moneer Gad / Engineering Research Journal 167 (September, 2020) A 124 - A 137

الأكو اد:

22. DIN - German Institute for Standardization (Deutsches Institut für Normung) 276 277. 